\title{
Musik als „Disciplin und Zuchtmeisterin“ ländlicher Untertanen in Sachsen (17./18. Jahrhundert)
}

\author{
von \\ MARTINA SCHATTKOWSKY
}

Im kursächsischen Dorf Krögis, im Herrschaftsbereich des in der Meißner Gegend gelegenen Rittergutes Schleinitz, ${ }^{1}$ fanden sich im Mai 1703 die Mitglieder einer „Music-Societät“ zusammen, um zu Erhaltung guter Zucht und Ordnung eine Reihe von Leges und gesetzen zu erarbeiten. ${ }^{2}$ Mitglieder dieser Sozietät waren neben dem Pfarrer und dem Schulmeister Dorfbewohner aus der Umgebung von Krögis, christliche Personen, wie es heißt, die sich trafen, um zu gewißßen Zeiten ibre musicalischen Zusammenkünfte zu halten, darbey nebst andern Auditoribus so der Music nicht kundig, dennoch aber als Music Liebhaber, in die Societät eingelaßen, in erbaulichen Gesprächen ergötzen, die edle Sing- und Klinge-Kunst, auf möglichste fleißigst exerciren. ${ }^{3}$

Auch im benachbarten Schleinitzer Kirchspiel Leuben existierte ein „Collegium Musicum“. ${ }^{4}$ Die Mitglieder dieses Kollegiums sind namentlich bekannt. Es waren dies die Pfarrer und Schulmeister aus Leuben und Ziegenhain sowie 14 Untertanen, die teils aus Dörfern des Schleinitzer Rittergutes, teils aus benachbarten Herrschaften kamen. Bei den sechs Schleinitzer Mitgliedern handelte es sich um einen Bauern, zwei Gärtner, zwei Leineweber und einen Böttcher. ${ }^{5}$

1 Vgl. zum Rittergut Schleinitz: Martina Schattkowsky, Zwischen Rittergut, Residenz und Reich. Die Lebenswelt des kursächsischen Landadligen Christoph von Loß auf Schleinitz (1574-1620) (Schriften zur sächsischen Geschichte und Volkskunde 20), Leipzig 2007. - Bei dem vorliegenden Aufsatz handelt es sich um eine überarbeitete Fassung eines Beitrags aus: Axel Lubinski/Thomas Rudert/Martina Schattkowsky (Hg.), Historie und Eigen-Sinn. Festschrift für Jan Peters zum 65. Geburtstag, Weimar 1997, S. 329-343.

2 Vgl. die Satzung dieser Sozietät von 1703 in: Sächsisches Staatsarchiv-Hauptstaatsarchiv Dresden (im Folgenden: HStA Dresden), Grundherrschaft (im Folgenden Gh.) Schleinitz, Nr. 1300. Wie diese Akte sind auch die Dokumente weiterer hier zitierter Schleinitzer Quellenbestände, falls nicht anders vermerkt, unpaginiert.

3 HStA Dresden, Gh. Schleinitz, Nr. 1300.

4 Vgl. dazu Pfarrarchiv Leuben (im Folgenden: PfA Leuben), Nr. 474: Collegium Musicum betr., 1704. Diese Akte enthält eine Satzung der Leubener Sozietät aus dem Jahr 1704 (im Folgenden: Satzung von 1704), eine undatierte zweite Fassung (im Folgenden: 2. Fassung) sowie Bruchstücke weiterer undatierter Entwürfe. Hinzu kommen Briefe, Eidesleistungen und Notizen über strittige Punkte der „Leges“. Ergänzt wird dieses Material durch Quellen des Schleinitzer Gutsarchivs, durch Landesordnungen sowie durch zeitgenössische musiktheoretische Arbeiten.

5 Die in den "Leges“ genannten Namen lassen sich anhand von Abgaben- und Dienstverzeichnissen der Schleinitzer Untertanen identifizieren. Vgl. z. B. HStA 
Dass körperlich schwer arbeitende Bauern und Dorfhandwerker um $1700 \mathrm{im}$ Rahmen eines Vereins nicht nur musizierten, sondern sich darüber hinaus in erbaulichen Gesprächen ergötzen wollten, mutet ungewöhnlich an. Hinzu kommt, dass zumindest die Leubener Musiksozietät damals keineswegs eine Neugründung war. In Leuben muss bereits um 1620 eine Musikvereinigung existiert haben, welche „in der Kirche bei währendem Gottesdienste aufwartete“ .6

Dies erscheint gleich in doppelter Hinsicht bemerkenswert: Einerseits widerspricht dies einmal mehr Auffassungen, wonach mit Blick auf die alltägliche Musikpraxis in protestantischen Gegenden im Vergleich zum katholischen Bereich eher eine kulturelle Ödnis unterstellt wurde.7 Gegen diesen defizitären Befund sprechen nicht allein vorliegende sächsische Beispiele, sondern ebenso neuere Studien zur musikalischen Praxis in ländlichen Gemeinden der Schweiz. ${ }^{8}$ Andererseits überrascht der frühe Zeitpunkt der genannten Quellenverweise auf „gehobene" Musikkultur in den Dörfern, die gemeinhin eher als Ergebnis der Aufklärung im 18. Jahrhundert zu vermuten wäre. ${ }^{9}$ Dabei waren kunstvoll musizierende Untertanen auf dem Lande ebenso wie die - von Jan Peters erwähnten - philosophierenden Bauern kein Phänomen erst des 19. Jahrhunderts, ${ }^{10}$ und es bedurfte ebenso wenig erst eines Engagements der Aufklärer des 18. Jahrhunderts, um diese Facette dörflichen Lebens zu entfalten.

\section{$I$.}

Gleichwohl würde man Musiksozietäten wie die in Krögis oder Leuben vor 1700 wohl eher im städtischen Bereich erwarten. Tatsächlich entstanden hier schon im

Dresden, Gh. Schleinitz, Nr. 968, Hand- und Spanndienste des Rittergutes Schleinitz 1639-1806.

6 Zitiert nach Franciscus Nagler, Das klingende Land. Musikalische Wanderungen und Wallfahrten in Sachsen, Leipzig 1936, S. 235. Auch Gustav Wilhelm Segnitz gibt Christoph von Loß als Gründer des „Collegiums“ an, ohne allerdings auf Quellen zu verweisen. Vgl. Gustav Wilhelm Segnitz, Einige geschichtliche Nachrichten über die Kirche und Kirchfahrt zu Leuben, Meißen 1839, S. 42.

7 Vgl. bezogen auf protestantische Orte in der Schweiz bei Peter Hersche, Gelassenheit und Lebensfreude. Was wir vom Barock lernen können, Freiburg im Breisgau 2011, S. 139-141.

8 Vgl. Daniel Schläppi, „Einige Zeit aufs Singen verwendet“. Musik als Kernressource dörflichen Kulturlebens am Beispiel des schweizerischen 18. Jahrhunderts, in: Zeitschrift für Agrargeschichte und Agrarsoziologie 64 (2016), H. 1, S. 11-35, hier S. 13.

9 In der Forschung wird gemeinhin das Beharrungsvermögen des traditionellen Bildes vom „rohen“, „ungebildeten“ Bauern betont und ein Wandel erst in die zweite Hälfte des aufgeklärten 18. Jahrhunderts verlegt. Vgl. Johann Ludewig, Der Gelehrte Bauer, Neudruck der ersten Ausgabe Dresden 1756, mit einem Nachwort von Holger Böning, Stuttgart/Bad Cannstatt 1992, hier S. 262 ff.

10 Vgl. Jan Peters, Zur Auskunftsfähigkeit von Selbstzeugnissen schreibender Bauern, in: Winfried Schulze (Hg.), Ego-Dokumente. Annäherung an den Menschen in der Geschichte (Selbstzeugnisse der Neuzeit 2), Berlin 1996, S. 186. 
16. Jahrhundert, anknüpfend an die Tradition mittelalterlicher Bruderschaften, zahlreiche musikalische Vereinigungen. $\mathrm{Zu}$ nennen sind etwa „Bürgerchöre“11 oder die Kantoreien, die bereits kurz nach der Reformation gerade auch in vielen sächsischen und thüringischen Städten zur Pflege des kirchlichen Kunstgesangs entstanden. ${ }^{12}$ Eine den Kantoreien nahestehende Vereinigung außerhalb von Kursachsen war z. B. die 1600 neu gegründete Musikantengilde im mecklenburgischen Friedland. ${ }^{13}$ Nach dem Dreißigjährigen Krieg breitete sich eine neue „Musikwelle" aus: Brachliegende Kantoreien wurden wiederbelebt und in zahlreichen sächsischen Städten sowie auch in anderen Gebieten richtete man "Collegia Musica" ein. ${ }^{14}$ Im Jahr 1653 gründeten mehr als einhundert (Rats-) Musikanten in 40 Städten zweier Reichskreise einen interlokalen Verband, das „instrumentalmusikalische Collegium in dem Ober- und Niedersächsischen Kreis". ${ }^{15}$ Darüber hinaus spielte der Musikunterricht in den Schulen eine wichtige Rolle in den städtischen Kirchenordnungen. ${ }^{16}$

Weitaus weniger weiß man über ländliche Musikvereine. Bekannt wurden in der Zeit des ausgehenden 17. Jahrhunderts z. B. die den städtischen Kantoreien nahestehenden „Adjuvantenvereine "17 in Thüringen oder sorbische Musikanteninnungen ${ }^{18}$. Im Altenburgischen sollen am Ende des 18. Jahrhunderts 40 wohl-

11 Beispiele dafür bringt Emil Sehling, wie z. B. die der fraternität oder bruderschaft des bürger chores der Stadt Münsterberg von 1577. Vgl. EmIL SEHLING, Die evangelischen Kirchenordnungen des XVI. Jahrhunderts, Bd. 3, Nachdruck Aalen 1970, S. 466-468. Im kursächsischen Raum existierte z. B. 1581 ein „Collegium Musicum“ in der Stadt Leisnig. Vgl. HStA Dresden, Landesregierung, Privilegien, Vol. XCI, fol. $776 \mathrm{ff}$.

12 Vgl. dazu Johannes Rautenstrauch, Luther und die Pflege der kirchlichen Musik in Sachsen (14.-19. Jahrhundert), Leipzig 1907, S. $118 \mathrm{f}$.

13 Vgl. ebd., S. 127 f.

14 Außerhalb von Sachsen entstanden Musikkollegien z. B. in Hamburg, Frankfurt oder Lübeck. Solche "Collegia Musica“ hatten, anders als die in erster Linie zur Ausschmückung des Gottesdienstes dienenden Kantoreien, mehr die Veranstaltung kirchlicher Konzerte im Auge. Vgl. Arno Werner, Geschichte der Kantorei-Gesellschaften im Gebiete des ehemaligen Kurfürstentums Sachsen (Publikationen der Internationalen Musikgesellschaft, Beiheft 9), Leipzig 1902, S. 19. Die hier zu untersuchenden Schleinitzer Musiksozietäten nahmen trotz gelegentlicher Bezeichnung als „Collegium musicum“ eher eine Zwischenstellung ein, da sie zwar auch konzertante Aufführungen darboten, zugleich jedoch wichtige Aufgaben bei Gottesdiensten zu erfüllen hatten.

15 Vgl. dazu Rudolf Wustmann, Sächsische Musikantenartikel (1653), in: NASG 29 (1908), S. 104-117, hier S. 104 f.

16 Vgl. etwa bei Sehling, Kirchenordnungen (wie Anm. 11), Bd. 3, S. 158, 219, 243, 405 sowie Bd. 1, S. 684, 703 f.; vgl. dazu auch Burkhard Busse, Zum Musikleben der Hansestadt Wismar im 17. Jahrhundert, in: Studien zur lokalen und territorialen Musikgeschichte Mecklenburgs und Pommerns 1 (1995), S. 81-88.

17 Während der städtische Chor nach der Bezeichnung seines Leiters, des Kantors, benannt wurde, verdankte der Adjuvantenverein seinen Namen dem Gehilfen des Schulmeisters, dem Adjuvanten. Vgl. Arno Werner, Freie Musikgemeinschaften alter Zeit im mitteldeutschen Raum (Schriftenreihe des Händelhauses in Halle 7), Wolfenbüttel/ Berlin 1940, S. 20.

18 Solche Musikanteninnungen entstanden vor allem dort, wo sich Rivalitäten zwischen sorbischen „Volksmusikanten“ und städtischen privilegierten Berufsmusikanten ent- 
habende Bauern alle 14 Tage in einem Schenkhaus Konzerte mit klassischer Musik gegeben haben. ${ }^{19}$ Ansonsten tauchen einzelne musizierende Dorfbewohner in der Literatur vor allem im Zusammenhang mit bäuerlichen Festen auf. Oft handelte es sich dabei um Tagelöhner oder andere besitzlose Dorfbewohner, die bei Hochzeiten der Bauern zum Tanz aufspielten. ${ }^{20}$ Dadurch gerieten sie nicht selten in Konkurrenzsituationen zu den Stadtpfeifern, für die Hochzeitsaufwartungen in Stadt und Land ein wichtiger Teil ihres Broterwerbs darstellten. ${ }^{21}$ Außerdem sind in diesem Zusammenhang fahrende Spielleute bzw. Musikanten zu erwähnen. Diese standen schon seit dem Mittelalter in einem unehrenhaften Ruf, den sie nur abwenden oder zumindest mindern konnten, indem sie sich einem christlichen Schutzpatron unterstellten und sich ebenfalls zu Musikergenossenschaften zusammenschlossen.22

In Landes- und Dorfordnungen ging die Obrigkeit gegen den Missbrauch der Musik durch niederträchtige Bierfiedler und Braten-Geiger vor, da viele darbey die Trunckenheit befördern und ibre Sinne verlieren. ${ }^{23}$ Wichtig war jedoch nicht nur der Erhalt des dörflichen Friedens, sondern auch die Erweiterung von herr-

wickelten. Leider sind keine Statuten dieser Innungen erhalten geblieben. Vgl. JaN RAupP, Sorbische Volksmusikanten und Musikinstrumente (Schriftenreihe des Instituts für sorbische Volksforschung 17), Bautzen 1963.

19 Vgl. ebd., S. 118.

20 Vgl. Dieter Krickeberg, Zur sozialen Stellung des deutschen Spielmanns im 17. und 18. Jahrhundert, besonders im Nordwesten, in: Walter Salmen (Hg.), Der Sozialstatus des Berufsmusikers vom 17. bis 19. Jahrhundert (Musikwissenschaftliche Arbeiten 24), Kassel u. a. 1971, S. 26-42, hier S. 31 f.; Jan Raupp bezeichnet für die Zeit des ausgehenden 17. Jahrhunderts den „armen Büdner“ als „Prototyp des sorbischen Volksmusikanten“. Vgl. Raupp, Sorbische Volksmusikanten (wie Anm. 18), S. 83.

21 Vgl. Johann Gottlob Klingners Sammlungen zum Dorf- und Bauren-Rechte, 3. Teil, Leipzig 1753, $\mathbb{S}$ CXXXI, S. 390 und $\$$ CXLI, S. 397. Aus den von Klingner zusammengestellten Fällen über die Bestellung von Musik aus der Zeit um 1700 geht hervor, dass Stadtpfeifer tatsächlich nicht nur für Musikaufführungen in Städten, sondern auch auf ländlichen Festlichkeiten herangezogen wurden. Aus zahlreichen Klageschriften wird ihr Bemühen deutlich, sich dieses Recht nicht durch „Pfuscher und Bier-Fiedler“ abspenstig machen zu lassen. Vgl. dazu auch Hermann Techritz, Sächsische Stadtpfeifer. Zur Geschichte des Stadtmusikwesens im ehemaligen Königreich Sachsen, Dresden 1932.

22 Vgl. dazu z. B. Richard van Dülmen, Der infame Mensch. Unehrliche Arbeit und soziale Ausgrenzung in der Frühen Neuzeit, in: Ders. (Hg.), Gesellschaft der Frühen Neuzeit: Kulturelles Handeln und sozialer Prozess (Kulturstudien. Bibliothek der Kulturgeschichte 28), Wien/Köln/Weimar 1993, S. 236-278, hier S. 245 ff.; sowie Walter Schatz, Die Zünfte der Spielleute und die Organisation der Orchestermusiker in Deutschland, rechts- und staatswissenschaftliche Dissertation Greifswald 1921, Teildruck Anklam 1921.

23 Vgl. Klingner, Sammlungen (wie Anm. 21), \ LX: Von Verpachtung der Musik, S. 344 ff.; auch unter den Schleinitzer Akten befindet sich ein Kirchenmandat aus dem Jahr 1626, in dem auf Üppigkeit und wildes Leben bei Lobetänzen und Gemeinden Bier verwiesen wurde. Auch verbot man darin angesichts der itzigen clemmen und schweren zeiten alle Täntze und spielleuthe, außer bei Hochzeiten und adelichen Versamblungen. HStA Dresden, Gh. Schleinitz, Nr. 1299, Kirchenmandat vom 10. März 1626. 
schaftlichen Machtkompetenzen, etwa durch das Recht, das Musizieren zu bestimmten Zeiten zu verbieten, oder durch die Beschränkung des Anspruchs der Dorfgemeinden auf eigene Auswahl von Musikanten. ${ }^{24}$ Johann Gottlob Klingner verweist in seinen „Sammlungen zum Dorf- und Bauren-Rechte“ auf eine ganze Reihe von Konfliktfällen wegen der von herrschaftlicher Seite angemaßten Verpachtung der Musik und Zwangs-Recht wider die Unterthanen. ${ }^{25}$ Gleichwohl erweist sich das herrschaftlich-bäuerliche Verhältnis in puncto Musikpflege keineswegs allein konfliktgeprägt.

\section{II.}

Die Schleinitzer Quellenbefunde erhärten den Eindruck, dass obrigkeitlicher Einfluss auf das dörfliche Musikleben bei den Untertanen durchaus auf eine positive Resonanz traf. Die herrschaftlicherseits betriebene Förderung von Kirchenmusik, der durchaus auch disziplinierende Absichten zugrunde lagen, nahmen die Dorfbewohner oft weniger als Zwang denn als Fundament für eigenständige kulturelle Praktiken wahr. ${ }^{26}$ Deutlich wird dies in ganz unterschiedlichen Belangen. So war etwa der Ausschluss fremder Spielleute auch für die Schleinitzer Musiksozietäten von großer Bedeutung. Im eigenen Interesse griffen die Mitglieder dabei auf die regulierenden und friedenserhaltenden Funktionen der Herrschaft zurück und erwarteten von ihrem Erb- und Gerichtsherrn sowie vom Pfarrer dahingehend Unterstützung, dass bei Hochzeiten oder anderen Ebren-Gelaken von Schleinitzer Untertanen nur Musikanten aus diesen Kollegien zugelassen würden. ${ }^{27}$

Wohlgemerkt handelte es sich bei diesen so selbstbewusst auftretenden Musikanten weder um „ungelehrte“ Dorfmusikanten ${ }^{28}$ noch um professionelle Spielleute, wie z. B. die bereits erwähnten Stadtpfeifer oder die herrschaftlichen „JagdPfeifer“,29 sondern um ländliche Untertanen, die selbst offenbar eine solche

24 Klingner, Sammlungen (wie Anm. 21), S. 347 f.

25 Ebd., S. 349, 392-396. Im Fall einer Streitigkeit zwischen dem Grafen von Schönburg zu Lichtenstein und den Einwohnern des Dorfes Oberlungwitz im Jahr 1710 wird dem Grafen vonseiten der Leipziger Juristenfakultät ausdrücklich untersagt, seine Untertanen an die sogenannten Jagd-Pfeifer zu weisen, und ibnen den Gebrauch anderer Spielleute zu inhibiren. Vgl. ebd., S. 388.

26 So auch bei Daniel Schläppi für die Schweiz; vgl. SchläppI, Musik als Kernressource (wie Anm. 8), S. 28.

27 Inwieweit zwischen beiden Musikvereinigungen Konkurrenzsituationen entstanden, lässt sich anhand der Quellen nicht feststellen. Allerdings scheint es schon wegen des Umfangs beider Kirchspiele genügend Betätigungsfelder für beide Kollegien gegeben zu haben. Immerhin gehörten zu Leuben 19 und zu Krögis 15 eingepfarrte Dörfer bzw. Dorfanteile.

28 Diesen Ruf hatten die Dorfmusikanten vor allem aus der Sicht der städtischen „Bildungsmusiker“. Vgl. so bei Krickeberg, Soziale Stellung (wie Anm. 20), S. 42. Tatsächlich aber unterschieden sie sich von den hier vorzustellenden Schleinitzer Musikanten, die sich in stärkerem Maße der Pflege der Kunstmusik verschrieben hatten. 
Professionalität erreichten, dass sie nicht nur Kirchenmusik aufführten oder auf bäuerlichen Hochzeiten und Begräbnissen spielten, sondern später sogar zu Festen ihrer Herrschaft auf das Schleinitzer Schloss sowie zu andern adeligen oder honorablen Ausrichtungen herbeigerufen wurden ${ }^{30}$ und bei diesen Anlässen eine Uniform oder Liverey aus Rock, Westen, Beinkleidern und Hutte mit silbernen Treßen ${ }^{31}$ trugen.

Die Gründung bzw. Neugestaltung der Schleinitzer Musikkollegien am Anfang des 17. und zu Beginn des 18. Jahrhunderts fiel jedes Mal in Zeiten wirtschaftlichen Aufschwungs, die sowohl den Rittergutsbesitzern als auch den Bewohnern des Untersuchungsgebietes günstige Einkommenschancen boten. Beide der damaligen Grundherren auf Schleinitz, Christoph von Loß (1574-1620) und Joachim Dietrich von Bose (1676-1742), haben ihre ökonomischen Spielräume gut genutzt und ergriffen vielfältige Maßnahmen zur Intensivierung ihrer auf Getreideverkauf orientierten Gutswirtschaft. ${ }^{32}$ Unübersehbar bemühten sich zudem diese Schleinitzer Herren, ihr Gut zu einem repräsentativen Adelssitz umzugestalten. Unter ihrer Herrschaft wurden das monumentale Wasserschloss und die dazugehörigen Neben- und Wirtschaftsgebäude renoviert bzw. umgebaut sowie Kunst- und Büchersammlungen erweitert. Große Aufmerksamkeit widmeten sie der Musikpflege. Christoph von Loß war den Quellen zufolge der Musica [...] wol kündig und ein sonderbarer liebbaber gewesen. ${ }^{33}$ Darüber hinaus hat der als strenger

30 Musizierende Bauern auf Festen des Landadels oder auch bei Hoffesten waren nichts Ungewöhnliches. Dabei hatte die Forschung allerdings vor allem Dorfmusikanten im Auge, die dort die lebensfrohe und entfesselnde Musik der Landbewohner vortrugen und so zur Bereicherung der zeremoniellen Formen etwa des Hoflebens beitragen sollten. Vgl. z. B. Krickeberg, Soziale Stellung (wie Anm. 20), S. 42. Hingegen gerieten Dorfmusikanten, die an Adelssitzen mit Kunstmusik aufwarteten, kaum einmal in das Blickfeld der Forschung.

31 Vgl. PfA Leuben, Nr. 474: Collegium Musicum betr., 1704, Extract aus denen von Hochadl. Gerichts-Herrschafft zu Schleinitz disfalls aufges. Puncten die Supernumerarios und Liverey betr., $\mathbb{S}$ 5. Diese Uniform durfte nur auf Anordnung der Schleinitzer Grundherren getragen werden, auf keinen Fall jedoch auf denen Dörffern und andern Orten oder in denen Schencken (vgl. ebd., $\ 8$ ). Dafür übernahm Joachim Dietrich von Bose die Kosten für die Uniform vollständig und erwartete von jedem Mitglied, dass es diese jederzeit fein reinlich und sauber halten soll (vgl. ebd., \5). Im Jahr 1783 hingegen hat der damalige Schleinitzer Rittergutsbesitzer Friedrich von Zehmen nur noch einen Teil der 13 Taler 11 Groschen 1 1⁄2 Pfennige pro Uniform (ohne die Leinwand) getragen, nämlich die Kosten für die Tressen auf den Hüten und an den Achselstücken. Aus der Kasse der Leubener Sozietät kam das Geld für Tuch, Unterfutter sowie die Knöpfe, und die Mitglieder übernahmen die Kosten für die Hüte, die Leinwand sowie den Macherlohn, was für jedes Mitglied ca. 5 Taler bedeutete (vgl. ebd., Brief vom 16. August 1783).

32 Vgl. Schatткоwsky, Zwischen Rittergut (wie Anm. 1), S. 82-92; sowie Dies., Loß (zu Schleinitz), Christoph d.J. von, in: Sächsische Biografie, hrsg. vom Institut für Sächsische Geschichte und Volkskunde e. V., bearb. von Ders., Online-Ausgabe: http:// saebi.isgv.de/ [Zugriff 9. August 2017].

33 Aegidius Strauch, Christliche Leichpredigt bey dem Begräbnüß Christoffen von Loß, Dresden 1620, unpag. (VD17: 14:051820K), gedruckt in: Schatтkowsky, Zwischen Rittergut (wie Anm. 1), S. 467-476; vgl. dazu auch Dies., „... und wolte ich mit ihnen in frieden und ruhe leben“. Hintergründe zum Herrschaftsverständnis adliger 
Lutheraner bekannte Loß sogar viel Christliche Gesenge selber gemacht und mit eigener Hand aufgeschrieben. ${ }^{34}$ Joachim Dietrich von Bose galt als Freund der Kunst und Wissenschaft, der am Ende des 17. Jahrhunderts in seinem Schlosspark ein Gartenhaus mit einer Bibliothek und einer Gemäldesammlung errichten ließ, wo auch Theateraufführungen stattfanden. ${ }^{35}$

Gerade hier zeigt sich die Vorbildwirkung des nur $50 \mathrm{~km}$ entfernten Dresdner Hofes, der bekanntlich die Entwicklung der Kirchen-, Opern- und Kammermusik dieser Zeit entscheidend mitgeprägt hat. ${ }^{36}$ Durch vielfältige familiäre Kontakte oder Ämtertätigkeit der Schleinitzer Rittergutsfamilien ergaben sich enge Verbindungen zur Residenzstadt. Auf diese Weise pflegte z. B. der Hofmarschall und Reichspfennigmeister Christoph von Loß in Dresden und Leipzig Umgang mit berühmten Musikern. Dazu zählte kein Geringerer als Heinrich Schütz. ${ }^{37}$ Doch gelangte „höfischer Glanz“ bisweilen auch direkt bis nach Schleinitz. Christoph von Loß konnte den Kurfürsten Christian II. mit dessen Gefolge gelegentlich zu Jagdvergnügen auf seinem Landgut empfangen. Zu Lebzeiten des von Bose fanden z. B. in Schleinitz und Umgebung um 1721 eine Reihe von Hoffestlichkeiten mit Parforcejagden und Theateraufführungen statt. ${ }^{38}$

Kulturelle Einflüsse der Residenz konnten aber ebenso durch ländliche Untertanen verbreitet werden. In der Leubener Satzung wurden beispielsweise die Mitglieder aufgefordert, mit berühmten Musico Bekandtschafft zu machen und zur Übung des musicalischen gehörs an Orte zu gehen, allwo die Music floriret. ${ }^{39}$ Erinnert sei in diesem Zusammenhang auch an die Einbeziehung von Bauern oder Bergleuten in das höfische Festwerk des 16. Jahrhunderts sowie später besonders

Rittergutsbesitzer in Kursachsen um 1600, in: Jan Peters (Hg.), Konflikt und Kontrolle in Gutsherrschaftsgesellschaften. Über Resistenz- und Herrschaftsverhalten in ländlichen Sozialgebilden der Frühen Neuzeit (Veröffentlichungen des Max-Planck-Instituts für Geschichte 120), Göttingen 1995, S. 359-403, hier besonders S. 379 ff.

Vgl. Strauch, Leichpredigt (wie Anm. 33).

35 Vgl. Отto Eduard Schmidt, Die Lommatzscher Pflege und das Geschlecht derer von Schleinitz (Kursächsische Streifzüge 3), Leipzig 1906, S. 100; sowie NAGLER, Das klingende Land (wie Anm. 6), S. 236.

36 Vgl. dazu Moritz Fürstenau, Zur Geschichte der Musik und des Theaters am Hofe zu Dresden, Dresden 1861, Nachdruck Leipzig u. a. 1971; sowie IRMgard BeCKERGlauch, Die Bedeutung der Musik für die Dresdener Hoffeste bis in die Zeit Augusts des Starken, Kassel/Basel 1951; Wolfram Steude, Zur Musik am sächsischen Hof in Dresden während der Regierung Kurfürst Johann Georgs II., in: Dresdner Hefte 33 (1993), S. 69-79; Matthias Herrmann (Hg.), Die Musikpflege in der evangelischen Schlosskapelle zu Dresden zur Schütz-Zeit (Sächsische Studien zur älteren Musikgeschichte 3), Altenburg 2009; sowie zuletzt Romy Petrick, Dresdens bürgerliches Musik- und Theaterleben im 18. Jahrhundert, Marburg 2011, besonders S. 37-46, 262267.

37 Vgl. den Brief von Heinrich Schütz an den Reichspfennigmeister Christoph von Loß vom 23. September 1616 in: Erich H. Müller (Hg.), Heinrich Schütz. Gesammelte Briefe und Schriften (Deutsche Musikbücherei 45), Regensburg 1931, S. 38 ff.

38 Vgl. dazu H. Zscherpel, Schloß Schleinitz bei Leuben, in: Döbelner Heimatschatz 2 (1923), S. 332-337, hier S. 335.

39 PfA Leuben, Nr. 474: Collegium Musicum betr., 1704, Satzung von 1704, $\$ 7$. 
in der Zeit Kurfürst Friedrich Augusts I. (des Starken). ${ }^{40}$ Volkskundliche Untersuchungen zeigen, wie sich um Dresden mit seinen Rats- und Amtsdörfern „eine Kontaktzone für Aufnahme und Weitergabe des höfischen Festwerks" bildete, indem die dortigen Dorfbewohner höfischen Veranstaltungsstil nachahmten und damit ein Vorbild schufen für von der Residenz entfernter gelegene Orte. ${ }^{41}$

Selbst wenn dies vielleicht Assoziationen weckt zu jenem von der Forschung gelegentlich als „Zirkulation kultureller Modelle“ beschriebenen Prozess, wobei höfische Kultur und Verhaltensweisen allmählich in andere soziale Schichten eindringen, ${ }^{42}$ war die Gründung der Schleinitzer Musiksozietäten alles andere als ein einseitig von der Herrschaft initiierter Akt kultureller Übertragung. Musste man doch allein schon bei den Dorfbewohnern entsprechende musikalische Interessen und Fähigkeiten vorausgesetzt haben können, wenn von ihnen erwartet wurde, in absehbarer Zeit eine gute Reputation bei der öffentlichen Darbietung von Vocal- und Instrumentalmusic zu erlangen und schließlich ihre Kunstfertigkeit bei berühmten Musikern zu steigern. Obendrein erschließt sich uns durch diese weithin unbeachtete Form ländlicher Musikpflege ein Ausschnitt sozialer Beziehungen, wo die daran beteiligten Obrigkeiten und Untertanen - ähnlich wie etwa bei herrschaftlich-bäuerlichen Auseinandersetzungen um „rein“ ökonomische Angelegenheiten - in einem „ständigen Prozeß von Kommunikation und Interaktion "43 um die Durchsetzung ihrer jeweiligen Interessen rangen.

Wie sehr die Dorfmusiker in diesen Gestaltungsprozess der Musikgesellschaften eingriffen, zeigt sich bereits bei der Abfassung der Satzungen aus dem frühen 18. Jahrhundert, die - wie auch viele der Statuten städtischer Kantoreien - als ein Produkt freier selbstständiger Erwägungen und Entschließungen vonseiten der Mitglieder herausgestellt und durch die Unterschrift sämtlicher Mitglieder bekräftigt wurden. Die „Leges“ von Krögis wurden, wie es hieß, von den Membra als

40 Vgl. dazu besonders FrIEDRICH SIEBER, Volk und volkstümliche Motivik im Festwerk des Barocks. Dargestellt an Dresdner Bildquellen (Veröffentlichungen des Instituts für Deutsche Volkskunde 21), Berlin 1960.

41 Ebd., S. 156. Als Beispiele für die Motivwanderung vom Hof auf das Land nennt Friedrich Sieber die Ausbreitung des am Fürstenhof gängigen Begriffs des „Ringelreiters“ oder das in Dörfern der südlichen Oberlausitz zu findende Spiel des „Ritterstechens".

42 Ausgehend von der Analyse der „höfischen Gesellschaft“ durch Norbert Elias bezieht Roger Chartier auch das Voranschreiten des Zivilisationsprozesses z. B. durch die Verfeinerung der Lebenskunst ein. Dabei warnt er zugleich davor, die von Elias beschriebene Zirkulation kultureller Modelle ausschließlich als einen Prozess zu betrachten, der von oben nach unten verlief. Vgl. Roger CHARTIER, Gesellschaftliche Figuration und Habitus. Norbert Elias und „Die höfische Gesellschaft", in: Ders. (Hg.), Die unvollendete Vergangenheit. Geschichte und die Macht der Weltauslegung, Frankfurt/Main 1992, S. 64 f.

43 Heide Wunder, Das Selbstverständliche denken. Ein Vorschlag zur vergleichenden Analyse ländlicher Gesellschaften in der Frühen Neuzeit, ausgehend vom „Modell ostelbischer Gutsherrschaft", in: Jan Peters (Hg.), Gutsherrschaft als soziales Modell. Vergleichende Betrachtungen zur Funktionsweise frühneuzeitlicher Agrargesellschaften (Historische Zeitschrift, Beihefte Neue Folge 18), München 1995, S. 23-49, hier S. 38. 
Anfänger dieser Sozietät constituiret und erst danach ihrem Erb- und Gerichtsherrn sowie dem Meißner Superintendenten zur Bestätigung vorgelegt. Im Leubener Fall wandten sich im August 1704 vier der Musikanten an ihren Herrn mit der Bitte, das mit einigen Legibus umbschrenkte Colegium Musicum zu konfirmieren.

Durchaus unterschiedlich manifestiert sich der Einfluss der geistlichen und weltlichen Obrigkeit auf die Ausgestaltung der Vereinsstatuten. Während etwa die Krögiser Satzung von 1703, insbesondere das mit Luther-Zitaten versehene Vorwort, von Anfang an stärker die Federführung des Schulmeisters bzw. Pfarrers erkennen lässt, fehlt dies in den verschiedenen Fassungen der Leubener „Leges“ ${ }^{44}$ Dafür zeigen sich im Leubener Beispiel größere Bestrebungen vonseiten des Schleinitzer Grundherrn von Bose, die Gründungssatzungen von 1704 in den Folgejahren zunehmend in seinem Interesse umzugestalten.

Damit rief er jedoch die zumeist wohlhabenden Landbewohner des Untersuchungsgebietes in der fruchtbaren Lommatzscher Pflege auf den Plan. Diese verfügten nicht nur über finanziellen Rückhalt u. a. für die Entrichtung von einem Taler als Eintrittsgebühr in die Musiksozietät oder für die Anschaffung der Instrumente, ${ }^{45}$ sondern auch über genügend Spielräume und vor allem Selbstbewusstsein, ${ }^{46}$ um sich (auch) in der Gestaltung ihrer kulturellen Aktivitäten nicht allein von herrschaftlichen Ansprüchen leiten zu lassen. Ihr Durchsetzungsvermögen bei der Verteidigung eigener Interessen zeigten die Leubener Musikanten insbesondere bei der jährlichen Aktualisierung der Satzung, wobei sie versuchten, einzelne ihrer Beneficiis und Freybeiten zu bewahren bzw. zu erweitern. Auf den Hauptzusammenkünften beratschlagten die Mitglieder, wo die Leges (welche iedesmabl öffentlich abzulesen) zu verbeßern wären. ${ }^{47}$ Danach wurden die Veränderungen dem Schleinitzer Herrn in schriftlicher Form zur Erörterung übergeben. Eine Abordnung der Sozietät verhandelte dann mit dem Herrn über die neuen Punkte, bis sie mit selbigen durchgängig zufrieden gewesen und diesfalls Angelöbnis gethan. ${ }^{48}$ Angesichts der intensiven Bemühungen der Mitglieder, herrschaft-

44 Die überlieferten Satzungen beider Schleinitzer Musiksozietäten weisen auch sonst durchaus Unterschiede auf. Die aus 16 Punkten bestehende Krögiser Ordnung regelt mehr die allgemeinen Rahmenbedingungen. Dagegen sind sowohl die Leubener Satzung von 1704 als auch die später ergänzten Fassungen wesentlich detaillierter und enthalten zuerst 21 und später 32 Punkte.

$45 \mathrm{Da}$ anfangs, wie es in der Leubener Satzung hieß, die Einkünffte dieser Gesellschafft sehr geringe wären, sollte zunächst jeder Musiker sein Musikinstrument von seinen eigenen Mitteln kaufen (vgl. ebd., $\$ 29)$. Später dann wurden die Instrumente aus der Kasse der Sozietät bezahlt. Über die Preise der Instrumente erfährt man aus der Krögiser Satzung, dass drei neue Violinen unter 6 Thaler nicht angeschafft werden können (vgl. HStA Dresden, Gh. Schleinitz, Nr. 1300, \$14).

$46 \mathrm{Zu}$ Beginn des 19. Jahrhunderts hießen die Bauern der Lommatzscher Pflege im Volksmund "Sammetbauern“, die „Hochzeits-, Kindtaufs- und Erntefeste“ feierten, denen die ehemalige ländliche Einfachbeit fremd wäre und die sich gar Equipagen halten würden. Vgl. Sснмidt, Lommatzscher Pflege (wie Anm. 35), S. 72 f.

47 HStA Dresden, Gh. Schleinitz, Nr. 1300, \$ 6.

48 PfA Leuben, Nr. 474: Collegium Musicum betr., 1704, Schreiben des Joachim Dietrich von Bose, o. J. 
liche Eingriffsmöglichkeiten etwa in finanzielle Angelegenheiten oder bei der Festlegung der Zahl der Mitglieder zu begrenzen, hat Joachim Dietrich von Bose immer wieder seine Rechte im Zusammenhang mit der Leubener Musiksozietät in Erinnerung rufen müssen. Wiederholt verwies er bei Erörterungen neuer Punkte ausdrücklich auf seine Befugnisse, nicht nur den Numerum Membrorum zu vermebren, einzuschrenken, mit denen ibnen verstatteten Beneficiis und Freybeiten [...] eine Anderung zu treffen, sondern selbst das Collegium nach meinem Gefallen und Belieben hinwieder zu revociren und aufzubeben. 49

Tatsächlich aber hatten die Schleinitzer Rittergutsbesitzer ebenso ein Interesse an der Erhaltung der Musikkollegien wie die Untertanen selbst. Eine große Rolle spielten dabei auf beiden Seiten finanzielle Erwägungen. Für die Musikanten aus den Schleinitzer Dörfern war der Eintritt in die Musiksozietäten zu Beginn des 18. Jahrhunderts auch mit materiellen Vergünstigungen verbunden. Das Musizieren erwies sich hier im wahrsten Sinne als „,verborgene Ökonomie“ 50 , die den beteiligten Bauern, Handwerkern oder Schulmeistern neben ihrer hauptberuflichen Tätigkeit zusätzliche Einnahmequellen brachte. Die wichtigsten Finanzressourcen der Leubener Sozietät stellten neben den Erträgen aus der Kollekte, die auf dem Chore in der Kirche zu Leuben an Sonn- und Festtagen zusammenkam, dem Säckel-Geld bey Hochzeit-Predigten ${ }^{51}$ sowie den Bußgeldern der Mitglieder ${ }^{52}$ vor allem die Einkünfte aus den Musikaufführungen dar. Diese zahlreichen musicalischen Aufwarttungen boten zugleich für den einzelnen Musikanten günstige Möglichkeiten des eigenen finanziellen Zugewinns. Jedes Mitglied hatte von der jeweiligen Aufführung einen festen Betrag in die Kasse der Sozietät einzuzahlen, während darüber hinaus erwirtschaftete Geldbeträge in ihre eigenen Taschen flossen. Besonders einträglich war das Musizieren auf Hochzeiten. In Krögis kamen pro Hochzeit 4 Groschen in die Kasse. In Leuben differierten die Beträge je nach sozialer Position des „Hochzeitshauses“53: Bei einer Hochzeit, wenn sie groß ist, hatte ein Mitglied 8 Groschen (im Fall einer Witwe 6 Groschen) und bei einer kleinen 4 Groschen zu erlegen. ${ }^{54}$ Mit wachsender Professionalität folgten auch

49 Ebd.

50 Vgl. Jan Peters, Märkische Lebenswelten. Gesellschaftsgeschichte der Herrschaft Plattenburg-Wilsnack, Prignitz 1550-1800 (Veröffentlichungen des Brandenburgischen Landeshauptarchivs 53), Berlin 2007, S. 244-251.

51 PfA Leuben, Nr. 474: Collegium Musicum betr., 1704, 2. Fassung, $\ 4$ und $\ 5$.

52 Aufstellungen über jährliche Einnahmen aus den Bußgeldern sind nicht überliefert. Die in den Satzungen aufgeführten strafbaren Vergehen bezogen sich vor allem auf versäumte Proben (1 bis 2 Groschen Strafe) oder das Zuspätkommen (von 3 Pfennigen bis zu 4 Groschen). Für vergessene Musikinstrumente auf den Proben waren 3 Pfennige, auf den Haupt Exercitien 1 Groschen zu zahlen. Ein Verstoß gegen das Gebot eines ehrbaren und sittsamen Verhaltens auf den Konventen wurde mit 3 Pfennigen, grobe Verbrechen mit 6 Pfennigen bestraft; Injurien, Schmeb-Worte, Zanck und Uneinigkeit kosteten 2 Groschen.

53 Auch in Städten unterschied man nach Hochzeiten verschiedener sozialer Stände. Vgl. Busse, Musikleben in Wismar (wie Anm. 16), S. 83.

54 PfA Leuben, Nr. 474: Collegium Musicum betr., 1704, Satzung von 1704, $\$ 25$. 
Aufführungen bei Hochzeiten adliger oder anderer hochgestellter Personen: Für eine herrschafftliche Hochzeit mussten 1 Taler und für eine andere honorable 8 Groschen zur Casse geliefert werden. ${ }^{55}$ Nach Jahresrechnungen der Leubener Sozietät aus den Jahren 1763 bis 1781 kam man auf durchschnittlich 15 bäuerliche Hochzeiten im Jahr; adlige Hochzeiten werden in diesen Jahren nicht erwähnt. ${ }^{56}$ Die auf Hochzeiten eingespielten Gelder brachten der Leubener Sozietät jährlich um die 3 Taler und damit ca. $20 \%$ der gesamten Jahreseinnahmen. ${ }^{57}$ Wie hoch der Gewinn der einzelnen Mitglieder bei solchen Festlichkeiten war, liegt im Dunkeln. Ebenso verborgen bleiben Gelder, die beim Aufspielen im Dorfkrug und anderen spontanen Geselligkeiten in die Taschen der Musikanten gekommen sein mögen. Entsprechende Verbote, der Kirchen gewidmete Instrumenta in Schenken zu gebrauchen, ${ }^{58}$ verweisen darauf, dass z. B. die Krögiser Musici ihre musikalischen Fähigkeiten auch bei solchen Gelegenheiten einsetzten. Auf jeden Fall war das Musizieren auf derartigen Festen für den Einzelnen finanziell einträglicher als die nicht unmittelbar entlohnten Auftritte in der Leubener Kirche. Nicht umsonst mussten sich die Musiker als Gegenleistung für die Vergünstigung, alle und iede Aufwarttungen zu baben, ausdrücklich zu regelmäßigen Darbietungen in der Kirche verpflichten. ${ }^{59}$ Gleichwohl profitierten letztlich auch die einzelnen Musiker von musikalischen Präsentationen im kirchlichen Rahmen. Abgesehen von dem wohl nicht zu unterschätzenden Prestigegewinn, der mit solchen öffentlichen Auftritten verbunden war, kamen von der Leubener Kirche bedeutende finanzielle Zuwendungen an die Sozietät insgesamt: Sowohl die Kirche als auch das Hospital in Leuben steuerten jährlich je 2 Taler und 2 Groschen bei. 60 Außerdem machten die in der Kirche gesammelten „Chor-Pfennige“ ca. 40 \% der gesamten Jahreseinkünfte der Sozietät aus. ${ }^{61}$

Aber auch für die Herren auf Schleinitz gab es gute Gründe dafür, bei Musikvorführungen in der Kirche und auf ihren Festen keine bezahlten „Stadtpfeifer“ oder eigene „Jagdpfeifer“ anzustellen, sondern vielmehr die musikalischen Ambitionen ihrer Untertanen zu fördern und schließlich zu Beginn des 18. Jahrhunderts Musikanten aus den Dörfern in ihr Schloss zu holen. Die Kostenfrage stellte sich besonders angesichts der Häufigkeit der Anlässe für musikalische Aufführungen. Nach der Leubener Satzung waren die Mitglieder der Sozietät verpflichtet, außer auf Hochzeiten und Begräbnissen alle 14 Tage und an jedem Feiertag, einschließlich der Erntezeiten, in der Kirche zu spielen. Eine aus professionellen Musikern bestehende Kapelle, etwa nach dem Vorbild der „Churfürstlich Sächsischen

55 Ebd., 2. Fassung, $\int 5$.

56 HStA Dresden, Gh. Schleinitz, Nr. 1341.

57 Ebd.

58 Ebd., $\int 16$.

59 PfA Leuben, Nr. 474: Collegium Musicum betr., 1704, Satzung von 1704, $\$ 27$.

60 HStA Dresden, Gh. Schleinitz, Nr. 1341.

61 Ebd. 
Kapelle" bei Hof,62 kam aus Kostengründen auf die Dauer wohl nicht in Frage. Die feste Anstellung von "Jagdpfeifern" wäre ebenfalls kostspieliger gewesen, da diese besser, als die Dorf-Spielleute, wollen tractiret, und vor ibre Mühe belohnet seyn. 63

Ein weiterer Vorteil von musicalischen banden mit eigenen Untertanen ergab sich für die Schleinitzer Herren aus der Möglichkeit, dadurch die Musikpflege stärker in eigenem Interesse zu beeinflussen und Kontrolle über die Musikanten auszuüben. Der Eintritt in die Sozietät vollzog sich nach genauen, rechtsverbindlichen Regeln. Die strengen Aufnahmebedingungen, die Eidesleistungen der Mitglieder oder die Sonderung der Mitglieder in seniores und iuniores erinnern ähnlich wie bei den Kantoreien mitunter an die Wurzeln städtischer Zunftordnungen. ${ }^{64}$ Das eigene Vermögen oder der Besitz galten als Kaution für die aus der Mitgliedschaft entstehenden Verpflichtungen (sub hypotheca bonorum). ${ }^{65}$ Der Bewerber gelobte nach der Verlesung der üblich gewesenen Gesetze und Gewohnheiten mit einem mündlichen und schriftlichen Eid die Einhaltung der "Leges“ und musste versprechen, sich auf Erfordern der gnädigen Herrschafft zu Schleinitz zur musicalischen auffwartung nicht weniger zur Kirchen Music und privat Exercitien gebübrend zu stellen. ${ }^{66}$ Ein Verlassen der Sozietät war nur in Ausnahmefällen möglich und kostete einen Taler. Niemand durfte sich obne gnugsame Uhrsachen [...] wieder [...] separieren und etwa nur solange als ibm beliebet darbey bleiben. ${ }^{67}$ Selbst ein Wegzug aus dem Schleinitzer Gerichtsbezirk oder aus benachbarten Gegenden beendete nicht automatisch die Zugehörigkeit zum Verein, sondern verpflichtete auch weiterhin zumindest zur Teilnahme an den jährlichen Zusammenkünften. Sollte ein Mitglied mit Gewallt sich von dieser Sozietät [...] reißen, würde man ibme bey seiner Obrigkeit deswegen belangen. ${ }^{68}$

Die Erhaltung effizienter Kontrollmechanismen lag auch im eigenen Interesse der Sozietäten. Dabei griffen sie selbst auf Instrumentarien obrigkeitlicher Regulierungspraxis zurück, wie sie sich vor allem in den zu Beginn des 18. Jahrhunderts längst herrschaftlich umgeformten Dorfordnungen oder in der Rügegerichtsbarkeit darboten. Ein dichtes Netz von Regelungen und ein detaillierter Bußgeldkatalog erlaubten den disziplinierenden Zugriff auf das Verhalten der Mitglieder und normierten ihre äußere Erscheinung. Im Vordergrund standen neben der musikalischen Befähigung ein guter Lebenswandel und ein friedliches und dienstfertiges Benebmen. ${ }^{69}$ Immerhin gehörten die 2 Groschen, die für Vergehen wie

62 Die Gehälter für diese Kapelle betrugen im Jahr 1697 insgesamt 4634 Taler. Vgl. Fürstenau, Geschichte der Musik (wie Anm. 36), Teil 2, S. 13 f.

63 Klingner, Sammlungen (wie Anm. 21), S. 387.

64 Vgl. Rautenstrauch, Luther und Musik (wie Anm. 12), S. 139 f.

65 HStA Dresden, Gh. Schleinitz, Nr. 1300, $\$ 10$.

66 PfA Leuben, Nr. 474: Collegium Musicum betr., 1704, Eid des Johann Christian aus Gohlis vom 5. Oktober 1733.

67 HStA Dresden, Gh. Schleinitz, Nr. 1300, $\mathbb{1} 10$.

68 Ebd., $\ 11$.

69 PfA Leuben, Nr. 474: Collegium Musicum betr., 1704, 2. Fassung, $\mathbb{} 7$. 
Injurien, Schmeh-Worte, Zanck und Uneinigkeit zu entrichten waren, $\mathrm{zu}$ den höchsten Geldstrafen der Soziäten. ${ }^{70}$ Großen Wert legte man ferner auf Zuverlässigkeit und Pünktlichkeit; beim Zuspätkommen auf den Proben erhöhte sich die Strafe alle 15 Minuten um 3 Pfennige bis maximal 2 Groschen. Bußgelder erwarteten jeden, der einen sorgsamen Umgang mit den Musikinstrumenten und Uniformen vermissen ließ oder der vergaß, sein Instrument und Notenmaterial vollständig mitzubringen. Jedes Mitglied war ferner verpflichtet, sich auf den Proben bzw. Aufführungen in seiner reinlichen Kleidung ${ }^{71}$ einzufinden, sich ebrbar und sitt$\operatorname{sam}^{72} \mathrm{zu}$ benehmen und die Trunkenheit zu meiden.

Über die Einhaltung dieser Regeln wachte ein Directorium der Sozietäten, zu welchem in Leuben außer dem Schulmeister der Pfarrer, die zwei Ältesten der Musiker sowie der Älteste der Auditores gehörten. Dieses Directorium verzeichnete die von den Mitgliedern angezeigten strafbaren Verbrechen und veranlasste gegebenenfalls auf den Hauptzusammenkünften eine Untersuchung einzelner Fälle. Schließlich mussten Verstöße gegen die Satzung nach der Art des Rügens der Herrschaft angezeigt werden, die somit als übergeordnete Kontrollinstanz fungierte.

Besonders wichtig war die Kontrolle der Musikanten, die außerhalb der Schleinitzer Gerichte wohnten. Ein ganze Reihe der Leubener Regelungen sollte den disziplinierenden Einfluss auf gerichtsfremde Mitglieder gewährleisten, etwa wenn diese nicht auf Proben oder Aufführungen erschienen oder keine Strafgelder zahlten. Deswegen mussten sie nach der Leubener Satzung Bürgen stellen, die im Fall eines Verstoßes gegen die „Leges“ der Sozietät die Bußgelder zu entrichten hatten, falls jene eine solche Zahlung verweigerten. ${ }^{73}$ Zum anderen befürchtete man wohl auch finanzielle Einbußen, denn - so wurde in der Satzung argumentiert - den Hauswirthen könnte dadurch Gelegenheit gegeben werden, bei den musicalischen Ausrichtungen die auswärtigen Musici nicht mitzuzählen, mit der Begründung, diese wären von frembden Gerichten. ${ }^{74}$ Daher erging schließlich an diejenigen, die im Schleinitzer Gebiet nicht ibr fener und Herdt haben, die Aufforderung, sich zu bemühen, daselbsten anzukauffen..$^{75}$ Überliefert ist das Beispiel des Johann Christian aus Gohlis, der bei seiner Aufnahme in das Leubener „Collegium Musicum“ im Jahr 1733 versprechen musste, er werde sich, da es die Herrschaft zu Schleinitz gerne sehen möchte, für die Dauer seiner Mitgliedschaft an einem dergl. Ort, wo es mir gefällig [...] niederlaßen. ${ }^{76}$

Der disziplinierende Einfluss der Musiksozietäten erschöpfte sich indes nicht allein in der Durchsetzung der in den Satzungen festgeschriebenen Verhaltens-

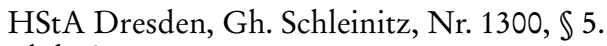

Ebd., $\$ 6$.

Ebd., $\$ 5$.

73 PfA Leuben, Nr. 474: Collegium Musicum betr., 1704, Satzung von 1704, $\mathbb{} 31$.

74 Ebd., $\$ 30$.

75 Ebd.

76 Ebd., Eid des Johann Christian aus Gohlis vom 5. Oktober 1733. 
maßstäbe und er beschränkte sich nicht ausschließlich auf ihre Mitglieder. Zu berücksichtigen sind darüber hinaus der ethische und ästhetische Wert der Musik selbst und die erzieherische Wirkung, die der Musik im damaligen Verständnis zugesprochen wurde. In der Vorrede der Krögiser Satzung von 1703 sah man in der Musik - in Anlehnung an Martin Luther - eine halbe Disciplin und Zuchtmeisterin, so die Leute gelinder, sanftmüthiger, sittsamer und vernünftiger machet. ${ }^{77} \mathrm{Im}$ Unterschied zur Schleinitzer Musikvereinigung des frühen 17. Jahrhunderts, die vorrangig der musikalischen Umrahmung des Gottesdienstes diente und offenbar vor allem auf Hebung der Kirchenzucht gerichtet war, trat zu Beginn des 18. Jahrhunderts stärker der Erziehungsgedanke im Hinblick auf eine „Verfeinerung der Sitten" sowie auch der Affektkontrolle hervor. Nach dem Verständnis der Verfasser der Schleinitzer Satzungen wurde der edlen Sing- und Klinge-Kunst eine Wirkung zugeschrieben, die die wildesten Gemüther zähmet, die erzörneten [...] erfreut. Daher sollte man, wie bereits von Luther gefordert, die Jugend stets zu dieser Kunst gewöhnen, denn sie machet feine geschickte Leute. Der Musik wurde gleich nach der Theologie der nechste Locum eingeräumt: Wer diese Kunst kann, der ist guter Arth und zu allen geschickt. Zur Untermauerung des Erziehungsgedankens berief man sich in den Krögiser „Leges“ u. a. auf den Pädagogen Valentin Trotzendorf (1490-1556), der als Rektor der Lateinschule in Goldberg (Złotoryja/Schlesien) ein gut durchorganisiertes Schulsystem geschaffen und dabei der musikalischen Bildung seiner Schüler einen großen Wert beigemessen hatte. ${ }^{78}$

Solche programmatischen Formulierungen in den Satzungen deckten sich im Übrigen auch mit anderen zeitgenössischen Ansichten über die von der Musik hervorgerufenen Emotionen und Einflüsse auf die menschliche Wesensart. Der Musiktheoretiker und Komponist Wolfgang Caspar Printz (1641-1717), dessen Werk „Historische Beschreibung der edelen Sing- und Kling-Kunst“"79 von 1690 sich auch in den Beständen der Schleinitzer Schlossbibliothek befand, ${ }^{80}$ unterschied zwei End-Ursachen der Musik: Eine Aeusserste und Gemeine sowie eine Aeussere und Eigene. ${ }^{81}$ Erstere bestand in der Ebre Gottes, letztere in der Bewegung des Menschlichen Gemüthes. ${ }^{82}$ Wichtig war auch hier der erzieherische Aspekt der Musik: Sie zöge die Gemüther der Zubörer hin, wohin sie will und würde jene formieren und sie geschlacht und wobl gemäßiget [...] machen..$^{33}$ An die Stelle eines wilden und ungebobelten Gemüths treten böfliche Sitten, eine leutsee-

77 HStA Dresden, Gh. Schleinitz, Nr. 1300. Die folgenden Zitate beziehen sich ebenfalls auf diese Quelle.

78 Vgl. z. B. das Stichwort „Trotzendorf“ in: Kurt Galling (Hg.), Die Religion in Geschichte und Gegenwart, Bd. 6, Tübingen ${ }^{3} 1962$, Sp. $1049 \mathrm{f}$.

79 Wolfgang Caspar Printz, Historische Beschreibung der edelen Sing- und KlingKunst, Dresden 1690, Nachdruck hrsg. von Othmar Wessely, Graz 1964.

80 PfA Leuben, Nr. 355: Die Schleinitzer Bibliothek betr., o. J.

81 Printz, Historische Beschreibung (wie Anm. 79), S. 170.

82 Ebd., S. 170, 173.

83 Ebd., S. 174. 
ligere und höfflichere Lebens-Art. ${ }^{84}$ Schließlich gelänge es mit Hilfe der Musik die Last der Arbeit zu mindern und zu versüssen, ${ }^{85}$ die Menschen zu trösten und Krankheiten zu heilen. 86

Inwieweit die Aufführungen der Schleinitzer Musikkollegien bei den Dorfbewohnern tatsächlich die gewünschten Wirkungen zeigten, lässt sich schwer beurteilen. Erschwerend kommt hinzu, dass uns die Quellen über das musikalische Repertoire der beiden Schleinitzer Musikvereinigungen im Unklaren lassen. ${ }^{87}$ Fest steht jedoch, dass die Ausstrahlung der Sozietäten im 18. Jahrhundert weit über den eigentlichen Kreis ihrer Mitglieder hinausreichte und auch deren Familien sowie eine steigende Zahl von Zuhörern regelmäßig mit Musik in Berührung brachte, sei es bei ihren Kirchenbesuchen, auf Begräbnissen oder auf den bereits erwähnten Hochzeitsfesten. Immerhin kam die Leubener Sozietät jährlich auf bis zu 17 Hochzeiten, die sich räumlich auf die 19 zu Leuben eingepfarrten Dörfer88 sowie auch darüber hinaus auf Ortschaften benachbarter Herrschaften erstreckten.

Eine starke Anziehungskraft entfalteten überdies die Proben und feierlichen Jahrestreffen der Sozietäten, die auch für Nichtmitglieder zugänglich waren. Um sich in Vocal und Instrumental-Music [zu] üben, traf man sich in Krögis alle drei Wochen, in Leuben alle vier Wochen jeweils donnerstags. Die Treffen fanden in der Zeit von 1 bzw. 2 Uhr nachmittags statt und dauerten etwa zwei Stunden; in Leuben gab es neben diesen kleinen Exercitien noch vierteljährlich Haupt Exercitien, die um 11 Uhr begannen und im Sommer um 6 bzw. im Winter um 4 Uhr endeten. In Erntezeiten fielen indes die Proben aus und wurden erst nach dem Erntedankfest wieder aufgenommen. Dass diese Probestunden nicht nur musikalischen Übungen dienten, sondern obendrein auch geselligen Charakter trugen, zeigen beispielweise die Jahresrechnungen der Leubener Sozietät, in denen die Kosten für das auf den Proben konsumierte Bier zu den größten Ausgabeposten gehörten. ${ }^{89}$

84 Ebd., S. 174 f.

85 Ebd., S. 192.

86 Die heilende Wirkung der Musik erklärte Printz damit, dass durch die von ihr hervorgerufenen Schwingungen eine dem Gemüth und Obren lieblich-fallende Harmoni erweckt würde. Die durch imprimierte Ton-Bewegung gleich bewegte Lufft dringe in den Leib und setze dort die Lebens-Geister in den Fäserlein, Häutlein oder FleischMäusen [Muskeln - M. Sch.] in Bewegung, die nun wiederum dazu führe, dass der Patient durch einen angenehmen Kützel und Anreitzung zum Tantzen und Springen getrieben wird. Bei der dadurch entstehenden Hitz und Erwärmung erweiterten sich die Lufft-Löchlein, durch welche die besonders in den Fleisch-Mäusen festgesetzten Gifte und scharffe Feuchtigkeit entweichen könnten. Ebd., S. 181.

87 In den Quellen klingt lediglich an, dass man sich um eine ständige Erweiterung des Programms bemühte. Der mit der musikalischen Leitung betraute Schulmeister sollte stets auf neue Stückgen und Parthien zur excolirung der Musique bedacht seyn. PfA Leuben, Nr. 474: Collegium Musicum betr., 1704, Satzung von 1704, $\$ 11$. Ebd. 
Probeort war im Krögiser Fall in der Regel die Schule, während man in der Leubener Schule nur zur Winterszeit zusammenkam. Im Sommer richtete der Schulmeister seine große Stube dazu ein, was im Winter zu viel Holz kosten würde. ${ }^{90}$ Gelegentlich fanden die Übungsstunden auch zu Hause bei den einzelnen Mitgliedern statt. Es spricht für die Wertschätzung der Arbeit der Leubener Sozietät, wenn die Musikanten in den 1730er-Jahren sogar im Schleinitzer Schloss proben durften. Sie trafen sich dort alle Monathe an einem Donnerstage, wobei deßentwegen jedesmabl darum anzufragen war. ${ }^{91}$

Das Interesse an der Arbeit der Sozietät, sei es als Mitglied oder als Zuhörer, war unter der Dorfbevölkerung der Schleinitzer Gegend offenbar so groß, dass die Begrenzung der Teilnehmerzahl bald eine wichtige Rolle in den Satzungen beider Kollegien spielte. Für die Vokalmusik ging man zur besseren Erfüllung der Kirche von vier bis sechs Musikern aus; für die Instrumentalmusik erachtete man sechs Personen als notwendig, denn außer dieser Zabl kein rechtes Chor gemacht werden kann. ${ }^{92}$ Mehr als acht Instrumentalisten durften aber nicht aufgenommen werden. Die Zahl der bereits erwähnten Auditores, die mit der Musique nichts zu schaffen haben, ${ }^{93}$ wurde ebenfalls auf acht begrenzt. Im Hinblick auf die Gäste bei Proben und geselligen Treffen nahm man in die Satzung auf, dass jedes Mitglied nicht mehr als einen Gast mitbringen durfte. Ausgenommen von solchen Beschränkungen blieben allein die eigenen Kinder der Musiker, welche des musiciren sich befleißigen wollen. ${ }^{94}$

Besonderer Beliebtheit erfreuten sich die Haupt-Zusammenkünffte der Sozietäten, die zunächst vierteljährlich stattfanden und in Leuben seit den 1730er-Jahren um mebrerer Beschwerlichkeit willen in Ansebung der Ausrichtung auf eine zu Michaelis stattfindende Zusammenkunft beschränkt wurden. ${ }^{95}$ Auf einem solchen Convent fanden sich auch die Familien der Mitglieder, die Auditores sowie weitere Gäste ein. In diesem Kreis besprach man wichtige Angelegenheiten der Sozietäten: Änderungen der Satzung wurden diskutiert, satzungswidrige Vergehen untersucht und gegebenenfalls neue Direktorien der Sozietäten gewählt. Hier erfolgte die Rechnungslegung der „Vereinsfinanzen“ durch den Leubener Schulmeister bzw. die beiden Vorsteher in Krögis.

Die Jahrestreffen waren zugleich gesellschaftliche Höhepunkte im Dorf. Die Leubener Musikanten trafen sich an diesem Tag um 9 Uhr vormittags. Mittags um 1 Uhr begann das gemeinsame Essen mit den Gästen, für das der Schulmeister zuständig war. Ein Blick auf den üppigen Speiseplan für einen solchen Tag vermit-

90 Vgl. PfA Leuben, Nr. 474: Collegium Musicum betr., 1704, Satzung von 1704, $\ 13$.

91 Ebd., 2. Fassung, $\$ 8$.

92 Ebd., Satzung von 1704, $\ 4$. Über die Instrumentalbesetzung der Sozietäten geben die Quellen keine genaueren Auskünfte. Im 18. Jahrhundert werden drei neue Violinen und die Reparatur einer Bassgeige erwähnt.

93 Ebd., $\$ 5$.

94 Ebd., 2. Fassung, $\mathbb{} 15$.

95 Ebd., $\ 13$. 
telt einen Eindruck von der guten Finanzlage der Sozietäten:96 In Leuben reichte man bei dieser Gelegenheit eine Suppe, gekochtes Fleisch, eine Schüssel Fische, einen Braten, Zugemüße, Butter und Käse, ${ }^{97}$ später kam noch eine Tonne Bier ${ }^{98}$ hinzu. Auf den viermaligen Treffen der Krögiser Sozietät sollten die Mitglieder jedes Mal, wenn der Fiscus so vermögend, ein Gericht Fleisch oder Fisch, iedoch beydes nicht zugleich, sowie Butter, Käse und Brot erhalten. ${ }^{99}$ Die Speisen der Mitglieder trugen die Kassen der Sozietäten. Das Bier hingegen musste jeder selbst bezahlen, ebenso 2 Groschen für das Essen jedes anwesenden Familienmitgliedes oder Gastes; die Auditores entrichteten für jeden Gast 4 Groschen.

\section{III.}

Über den weiteren Verlauf solcher Treffen schweigen die Schleinitzer Quellen. Auch wenn sicherlich mit fortschreitender Tageszeit und unter dem Eindruck reichlichen Biergenusses die eingangs erwähnten erbaulichen Gespräche allmählich in den Hintergrund getreten sein mögen, wurde doch deutlich, dass die Pflege der Sing- und Klingekunst durch die Schleinitzer Musikanten weit über das hinausging, was man nach landläufigen Vorstellungen mit dem Musizieren im Dorf und mit bäuerlichen Festen verbindet, die sich demnach allein in Tanz, Trunk und Raufereien erschöpft hätten. Kunstmusik übende und ausübende Dorfbewohner hingegen finden in diesem Zusammenhang kaum einen Platz. Dabei waren kunstvoll musizierende Untertanen auf dem Lande kein Phänomen erst der Aufklärung. Für den in den ersten Jahren des 18. Jahrhunderts erhobenen Anspruch in den Satzungen der Sozietäten, durch Musik feine geschickte Leute zu erziehen, war damals offenbar schon der Boden bereitet. Indem man Dorfbewohner als Musikanten zu Darbietungen oder Probestunden in das Schleinitzer Schloss einließ, wurden kulturelle Grenzen zwischen der Herrschaft und ihren bäuerlichen Untertanen durchlässiger, wurden die Bauern und Dorfhandwerker in diesem Falle sozusagen „salonfähig“.

Die Erklärungsmöglichkeiten für diesen Prozess sind vielschichtig. Eine wichtige Rolle spielte sicherlich die lange musikalische Tradition, auf die die Schleinitzer Musikgesellschaften des beginnenden 18. Jahrhunderts aufbauen konnten. $\mathrm{Zu}$ verweisen ist nicht nur auf die fast einhundert Jahre zurückreichenden Wurzeln des Leubener Musikvereins selbst, sondern auch auf die zahlreichen, bereits gegen Ende des 16. Jahrhunderts gegründeten Kantoreien in benachbarten Städten. ${ }^{100}$

\footnotetext{
96 Die Jahresrechnungen der Leubener Sozietät von 1763 bis 1781 weisen ein Vermögen zwischen 52 und 80 Talern aus. HStA Dresden, Gh. Schleinitz, Nr. 1341.

97 PfA Leuben, Nr. 474: Collegium Musicum betr., 1704, Satzung von 1704, $\$ 23$.

98 Ebd., 2. Fassung, $\mathbb{S} 14$.

99 HStA Dresden, Gh. Schleinitz, Nr. 1300, $\ 4$.

100 So z. B. die in unmittelbarer Nachbarschaft von Leuben und Schleinitz liegende Kantorei in Lommatzsch (1570) oder die Kantoreien in Mügeln (1571), Roßwein (1567)
} 
Hinzu kommen die Residenznähe des Schleinitzer Gutes sowie die für Herrschaft und Bauern günstigen wirtschaftlichen Entwicklungsbedingungen. Dies beeinflusste auch die kulturellen Eigenheiten des Untersuchungsgebietes. Bereits in der älteren Forschungsliteratur philosophierte man über das bei den Bauern der Lommatzscher Pflege vergleichsweise wenig ausgeprägte "Altväterische“ in „Sitte, Tracht, Hausrat" oder im Hinblick auf die Volksliedtradition. ${ }^{101}$ Wichtige Gründe dafür sah man insbesondere im Wohlstand der Bauern, der eher den Weg freigemacht hätte für „die Genüsse und die Mode des Tages“.102 Das zeitige Eindringen „städtischen Wesens“ sowie der Hang der großen bäuerlichen Hufenbesitzer, es - „bei allem Respekt“ - dem adligen Rittergutsbesitzer gleichzutun, prägten nach dieser Ansicht schon früh die „ländliche Eigenart“. 103

Solche Überlegungen sind im Zusammenhang mit den Schleinitzer Musiksozietäten nicht von der Hand zu weisen: Die Orientierung ihrer Verfassung an städtischen Zunft- bzw. Kantoreiordnungen einerseits sowie die Anziehungskraft herrschaftlicher Lebensformen und Wertvorstellungen andererseits waren nicht $\mathrm{zu}$ übersehen. Immerhin wurde die gleiche Musikgruppe, die etwa auf adligen Hochzeiten spielte, auch für bäuerliche Feste engagiert. Außerdem weisen manche der durch die musikalischen Darbietungen intendierten Erziehungseffekte durchaus Parallelen zu Richtlinien in adligen Tugendkatalogen auf, wo eine „Verfeinerung der Sitten" und die Kontrolle der Affekte ebenso an zentraler Stelle zu finden waren. 104

Die Gründung solch anspruchsvoller ländlicher Musikvereine kam nicht zuletzt auch dadurch zustande, dass sie eine Reihe von Vorzügen bot, von denen nicht nur die Mitglieder selbst, sondern auch die Rittergutsfamilien auf Schleinitz profitieren konnten. Abgesehen vom finanziellen Nutzen einer eigenen Musikantenvereinigung, die noch dazu nach Belieben einsetzbar war, dürfte für die Schleinitzer Herrschaft das Bemühen um eine Disziplinierung ihrer Untertanen in Richtung auf Gehorsam, Pünktlichkeit und Friedfertigkeit durchaus von einigem Interesse gewesen sein. Verlockend war für die Rittergutsbesitzer möglicherweise ebenso die Vorstellung, Musik als Disciplin und Zuchtmeisterin im Sinne einer Erziehung der Dorfbewohner zu feinen geschickten Leuten einsetzen zu können. Nicht von Untertanen mit - wie oben erwähnt - groben und ungebobelten Gemüthern umgeben zu sein, sondern statt dessen etwa den Besuchern auf Schloss Schleinitz Bauern und Handwerker mit höfflicher Lebens-Art vorzuzeigen, konnte, verstärkt durch den Einfluss frühaufklärerischen und pietistischen Ge-

und Döbeln, deren Ursprünge sich sogar bis in das Jahr 1451 zurückverfolgen lassen. Vgl. die Übersicht bei Werner, Kantorei-Gesellschaften (wie Anm. 14), S. 15 ff.

101 Vgl. Reinhold Herrmann, Städter und Dörfler der Lommatzscher Pflege am Ausgange des 18. Jahrhunderts im Urteile eines Zeitgenossen, in: Döbelner Heimatschatz 3 (1924), S. 175-190, hier S. 188.

102 Ebd.

103 Ebd.

104 Vgl. dazu Schatткowsky, Herrschaftsverständnis (wie Anm. 33), S. 386 ff. 
dankenguts an der Wende vom 17. zum 18. Jahrhundert, mit dazu beigetragen haben, das Sozialprestige einer Landadelsfamilie zu erhöhen.

Dass wichtige Impulse für die Ausgestaltung des Schleinitzer Musiklebens im 18. Jahrhundert nicht allein von der Herrschaft, sondern auch von den musizierenden Dorfbewohnern selbst kamen, macht diesen Fall nur umso bemerkenswerter. Zwar konnte dieser Prozess nicht ohne die Förderung und Zustimmung der kirchlichen und weltlichen Obrigkeit erfolgen, doch waren der Gestaltungswille und die Gestaltungsfähigkeit der Untertanen in diesem speziellen Handlungsfeld unübersehbar: Ausschlaggebend waren nicht ausschließlich Disziplinierungsambitionen vonseiten der Schleinitzer Herrschaft, sondern ebenso die von den dörflichen Musikanten erhobenen Ansprüche auf Selbstdisziplinierung.

Das weitere Schicksal der Krögiser und Leubener Musikkollegien über die Mitte des 18. Jahrhunderts hinaus verliert sich im Dunkeln der Quellen. Noch einmal wird im Jahr 1839 ein herrschaftliches „Schleinitzer Musikkorps“ erwähnt. ${ }^{105}$ Inwiefern diese Kapelle noch etwas gemeinsam hatte mit den Musiksozietäten des 18. Jahrhunderts, muss weiteren Forschungen vorbehalten bleiben.

105 Vgl. Segnitz, Kirche zu Leuben (wie Anm. 6), S. 42. 